\title{
Stereomicroscopic Evaluation of Fiber Post Failure Modes after Use of Three Different Endodontic Sealers: An In Vitro Study
}

Anjaneya Shiva Prasad ${ }^{1}$, Ravi Vaiyapuri ${ }^{2}$, Ragavi Shanmugam ${ }^{3}$, Jambai Sampath Kumar Sivakumar ${ }^{4}$, Saravana Priyan Soundappan $^{5}$, Chittrarasu Mathimaraiselvan ${ }^{6}$

\begin{abstract}
Aim and objective: The aim and objective of this study was to evaluate the three different root canal sealers that influenced the failure modes of fiber post luted with self-adhesive resin cement. Under a stereomicroscope, the influence of three different endodontic sealers on the failure modes of fiber post luted with self-adhesive resin cement was evaluated and compared.

Materials and methods: In this study, 45 mandibular premolars were tested. To standardize the root length, the samples were decoronated at the cementoenamel junction. Working length was determined using the Ingles method. The samples were separated into three groups after cleaning and shaping, and each group was obturated with gutta-percha and the respective sealer. For 1 week, all of the samples were incubated at $37^{\circ} \mathrm{C}$. Drills given by the manufacturer were used to prepare the post area, and the appropriate post was luted with self-adhesive resin cement. The samples were incubated at $37^{\circ} \mathrm{C}$ for 1 week. Following that, the samples were sectioned into 2 -mm-thick slices at the coronal, middle, and apical levels. The universal testing machine was used to execute the push-out test, and the mode of failure was investigated under a stereomicroscope.

Result: Bio-C sealer group showed maximum mixed failure. Maximum cohesive failure was observed in the AH Plus group, and maximum adhesive failure was observed in the mineral trioxide aggregate (MTA) Fillapex group.

Conclusion: The group where the Bio-C sealer was used showed more amounts of mixed failure and better bond strength in comparison with AH Plus sealer and MTA Fillapex sealer.

Keywords: Bond strength, Endodontic sealer, Fiber post, Resin cement.

Journal of Operative Dentistry and Endodontics (2021): 10.5005/jp-journals-10047-0110
\end{abstract}

\section{INTRODUCTION}

The selection of an appropriate repair for natural function and esthetic rehabilitation in compromised teeth is a key problem. ${ }^{1}$ When the crown is clinically close to being damaged, intraradicular posts are recommended to provide retention between the prosthetic crown and the remaining tooth structure. ${ }^{2,3}$ This retention is critical to the long-term longevity of the ultimate repair. ${ }^{4}$

Fiber-reinforced composite posts have seen increased use in recent years ${ }^{5}$ because of advantages such as acceptable aesthetics, a modulus of elasticity similar to dentin, stress dispersion across a wider surface area on root canal walls, and a minimal risk of vertical root fracture. ${ }^{6,7}$ However, a variety of circumstances have been observed to obstruct the bonding of fiber posts to root dentin. The type of root canal sealer used during obturation is one of these variables.

Root canal sealers that have traditionally been used include zinc oxide eugenol, calcium hydroxide, and resin-based sealers. Newer root canal sealers are currently been developed to overcome the drawback of existing sealers. ${ }^{8}$

Bioceramics are ceramic materials used to treat and replace severely ill musculoskeletal system components. Bio- $C$ sealer, a calcium silicate-based sealer, was developed as a new generation endodontic sealer and is available in both premixed paste and powder liquid forms. Zirconium oxide, calcium silicates, calcium phosphate monobase, calcium hydroxide, and thickening agents were also present. ${ }^{9}$

Mineral trioxide aggregate (MTA) Fillapex is a sealer based on MTA. MTA Fillapex is a sealer that composed of MTA, salicylate
1,2,4-6Department of Conservative Dentistry and Endodontics, Vivekanandha Dental College for Women, Tiruchengode, Tamil Nadu, India

${ }^{3}$ Department of Conservative Dentistry and Endodontics, Vivekanandha Dental College, Thanjavur, Tamil Nadu, India

Corresponding Author: Ragavi Shanmugam, Department of Conservative Dentistry and Endodontics, Vivekanandha Dental College, Thanjavur, Tamil Nadu, India, Phone: +91 7395826510, e-mail: ragavis254@gmail.com

How to cite this article: Prasad AS, Vaiyapuri R, Shanmugam R, et al. Stereomicroscopic Evaluation of Fiber Post Failure Modes after Use of Three Different Endodontic Sealers: An In Vitro Study. J Oper Dent Endod 2021;6(1):6-10.

Source of support: Nil

Conflict of interest: None

resin, natural resin, bismuth oxide and silica. It is biocompatible and radiopaque, stimulates mineralization, also releases calcium ions, and exhibits good adhesive properties to dentin as well as sealing ability comparable to epoxy resin-based sealers. ${ }^{10}$

AH Plus is a two-component epoxy-amine resin-based paste/ paste root canal sealant. AH Plus is a hydrophobic epoxy resinbased sealer that has been considered as a gold standard in sealer comparisons. In this study, it has been used as a reference material for comparison. ${ }^{11}$ This study aims at determining the effect of MTA 
Fillapex and Bio-C sealers on the bonding efficacy of fiber post to radicular dentin.

The null hypothesis was that there would be no difference in the failure mode of fiber post luted with self-adhesive resin cement between these three different root canal sealers.

\section{Aim and Objective}

The aim of this study was to evaluate the three different root canal sealers that influenced the failure modes of fiber post luted with self-adhesive resin cement.

The objective of this study was to use a stereomicroscope to evaluate and compare the failure modes of fiber post luted with selfadhesive resin cement after using three different endodontic sealers.

\section{Materials and Methods}

\section{Ethical Clearance}

A protocol explaining the study's goal and procedures was submitted to the Institutional Review Board of Vivekanandha Dental College for Women and the study was approved.

\section{Methodology}

This study included 45 mandibular single-rooted premolars with mature apices extracted for therapeutic reasons. Cracked, carious, restored, or fractured roots were eliminated from the study. The samples were then stored in normal saline after being decoronated at the cementoenamel junction to standardize the root length. A size 10 stainless steel endodontic $K$-file was used to negotiate the root canal until the tip of the file was seen at the apical foramen. Radiographs were taken to confirm the working length, which was calculated by subtracting $1 \mathrm{~mm}$ from the initial length. Using ProTaper Gold rotary files, all of the canals were instrumented to the working length.

Throughout instrumentation, canals were irrigated with a $3 \%$ sodium hypochlorite solution and normal saline. For the final irrigation, $5 \mathrm{~mL}$ of $17 \%$ ethylenediaminetetraacetic acid was used for 1 minute, followed by $5 \mathrm{~mL}$ of normal saline. Following that, the samples were stored in normal saline and separated into three groups of 15 specimens each, based on the sealer used:

MTA Fillapex (Angelus) in group I

AH Plus (Dentsply) in group II, and

Bio-C (Angelus) in group III.

The sealer was mixed and injected into the canal using a selfmixing tip attached to a syringe provided by the manufacturer in group I (MTA Fillapex). The canal was then sealed with MTA Fillapex sealer and a size 25/0.06 GP cone was inserted until the working length was obtained. At orifice level, the remaining gutta-percha was sheared off.

The AH Plus sealer was mixed on a paper pad in group II ( $\mathrm{AH}$ Plus). A gutta-percha cone with a \#25/0.06 taper was coated with $\mathrm{AH}$ Plus sealer and pumped into the root canal a few times. The cone was taken out, resealed, and reinserted up to the working length. At orifice level, the remaining gutta-percha was sheared off.

The premixed bioceramic sealer is put into the canal with the syringe tip provided by the manufacturer in group III (Bio-C sealer). The sealer-coated gutta-percha was introduced into the root canal until it reached the working length, after which the remaining gutta-percha was sheared off at the orifice level.

Temporary cement was used to seal the coronal parts of canals (Cavit). For 1 week, all of the samples were incubated at $37^{\circ} \mathrm{C}$. Drills provided by the manufacturer were used to prepare the post space to a length of $10 \mathrm{~mm}$. The root canals were cleaned with distilled water, then dried with paper points before being luted with self-adhesive resin cement (Multilink). The samples were kept at $37^{\circ} \mathrm{C}$ for another week. Following that, the samples were cut into $2-\mathrm{mL}$ slices at the coronal, middle, and apical thirds of the post length.

A universal testing machine with a crosshead speed of $1 \mathrm{~mm} /$ minute was used to perform the push-out test on each specimen. The plunger used to have a diameter that was around (at least) $80 \%$ of the canal's diameter. Each specimen was examined under a stereomicroscope at a magnification of 40 times to determine the failure mode.

\section{Statistical Analysis}

The data were statistically examined using SPSS software version18.0, and the groups were compared using the Chi-square test.

\section{Result}

Figure 1 showed that intergroup comparsion of modes of failures among three group are represented by bar graph.

Adhesive failure was seen mostly in MTA Fillapex sealer (group I) when compared to AH Plus (group II) and Bio-C sealers (group III).

Cohesive failure was seen mostly in AH Plus sealer (group II) when compared to MTA Fillapex (group I) and Bio-C sealers (group III) sealers.

Mixed failure was seen mostly in Bio-C sealer (group III) when compared to MTA Fillapex (group I) and AH Plus (group II) sealers.

Table 1 shows that $60 \%$ of adhesive failures is more in MTA Fillapex when compared with $\mathrm{AH}$ Plus and Bio-C sealer.

$53 \%$ of Cohesive failures is more in $\mathrm{AH}$ plus when compared with MTA Fillapex and Bio C sealer.

$53 \%$ of Mixed failures is more in Bio- $C$ when compared with MTA Fillapex and AH Plus sealer. The statistical significance level was set at $<p 0.01$.

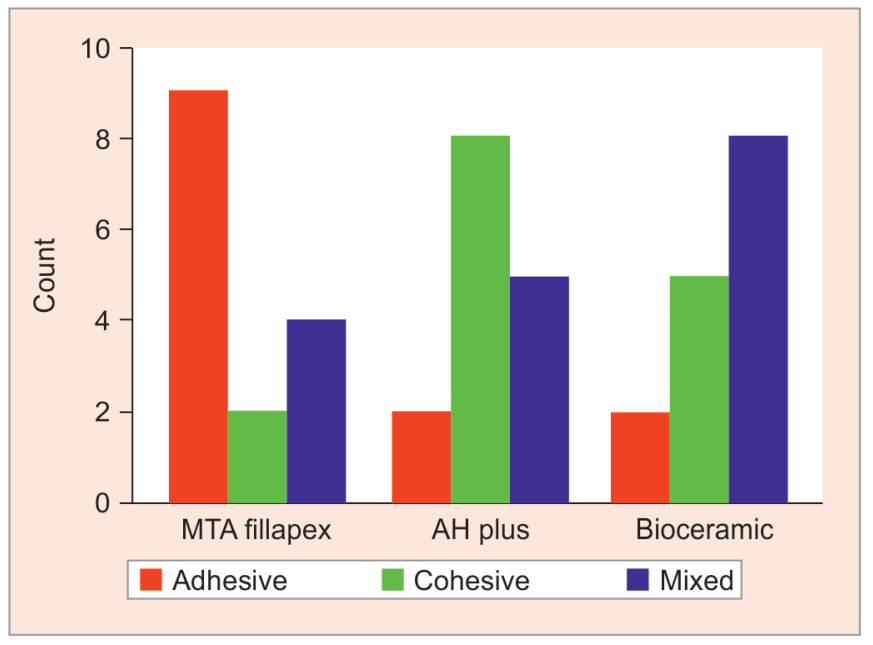

Fig. 1: Intergroup comparison of modes of failure among three groups

\section{Discussion}

Endodontic therapy aims to not only clean and shape the root canal to remove bacteria but also to assure that the root canal system 
Table 1: Mean value for the modes of failure among three groups using Chi-square test

\begin{tabular}{|c|c|c|c|c|c|c|c|c|c|}
\hline \multirow[b]{3}{*}{ Group } & \multicolumn{6}{|c|}{ Coronal } & \multirow[b]{3}{*}{ Total } & \multirow[b]{3}{*}{ Chi-square } & \multirow[b]{3}{*}{$p$} \\
\hline & \multicolumn{2}{|c|}{ Adhesive } & \multicolumn{2}{|c|}{ Cohesive } & \multicolumn{2}{|c|}{ Mixed } & & & \\
\hline & $N$ & $\%$ & $N$ & $\%$ & $N$ & $\%$ & & & \\
\hline MTA Fillapex & 9 & 60.00 & 2 & 13.33 & 4 & 26.67 & 15 & 12.67 & $0.013^{*}$ \\
\hline AH Plus & 2 & 13.33 & 8 & 53.33 & 5 & 33.33 & 15 & & \\
\hline Bioceramic & 2 & 13.33 & 5 & 33.33 & 8 & 53.33 & 15 & & \\
\hline Total & 13 & 28.89 & 15 & 33.33 & 17 & 37.78 & 45 & & \\
\hline
\end{tabular}

closes fluid tightly. Sealers have been considered essential since gutta-percha adheres poorly to canal walls. The main purpose of a root canal sealer is to fill up defects and improve the root filling material's adaptability to the canal walls. ${ }^{12-15}$

However, in teeth with significant coronal tooth structure loss, intraradicular posts may be necessary for final restorative support and retention.

Due to benefits like the ability to bind to the tooth structure, a modulus of elasticity similar to dentin, and enhanced aesthetics, prefabricated glass-fiber-reinforced composite posts have been popular in recent years. Fiber post cementing can be done with either traditional dual-cured resin cement or self-adhesive resin cement. Fiber posts cemented using self-adhesive resin cement have been found to have similar retention to posts cemented with traditional dual-cured resin cement. ${ }^{16-19}$

According to several studies, sealers have an effect on the bonding efficiency of fiber post luted to the root dentin with self-adhesive resin cement. As a result, we examined the bonding efficiency of self-adhesive resin cement with root dentin after using three different endodontic sealers in this study.

The push-out test simulates clinical conditions by applying shearing force to the dentin-post-cement surfaces. Because there is no premature failure, the push-out test offers a more adequate evaluation of resistance to dislodgment. ${ }^{20}$

The following criteria were used to categorize the failure modes: (Fig. 2)

Type I-Adhesive failure between dentin and resin cement.

Type II-Cohesive failure inside the dentin or within the cement. (Fig. 3)

Type III-Mixed failures, in which the sample showed both adhesive and cohesive failure. ${ }^{21}$ (Fig. 4)

The AH Plus sealer is an epoxy-bisphenol resin-based sealer made up of epoxy resin and amines. The epoxy resin interacts with the dentin collagen, creating a covalent bond with it, increasing the bond strength of this sealer to the root dentin. It has greater penetration into the microdefects in radicular dentin due to its flowability, creep capacity, and longer setting time, which enhances mechanical interlocking between sealer and root dentin.

The resin luting cement used in this study, Multilink, is a combination of hydrophilic and hydrophobic monomers. The later molecules will give hydrophobic characteristics, which are necessary to manage the moisture state of dentin and prevent the absorption of excess water, which might inhibit the polymerization process of the material; as a result, it will bind effectively with the epoxy resin-based sealer. ${ }^{22}$

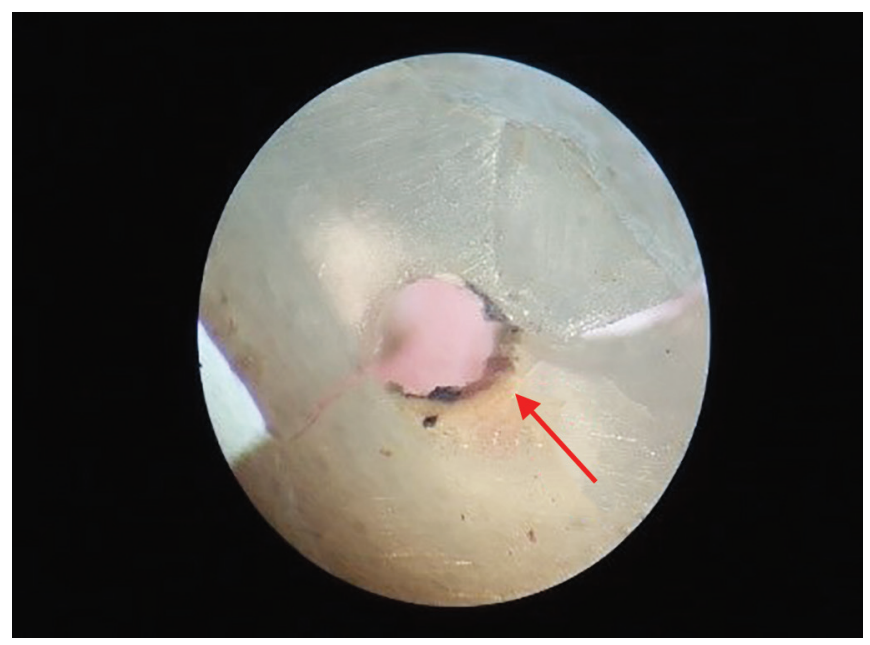

Fig. 2: Adhesive failure

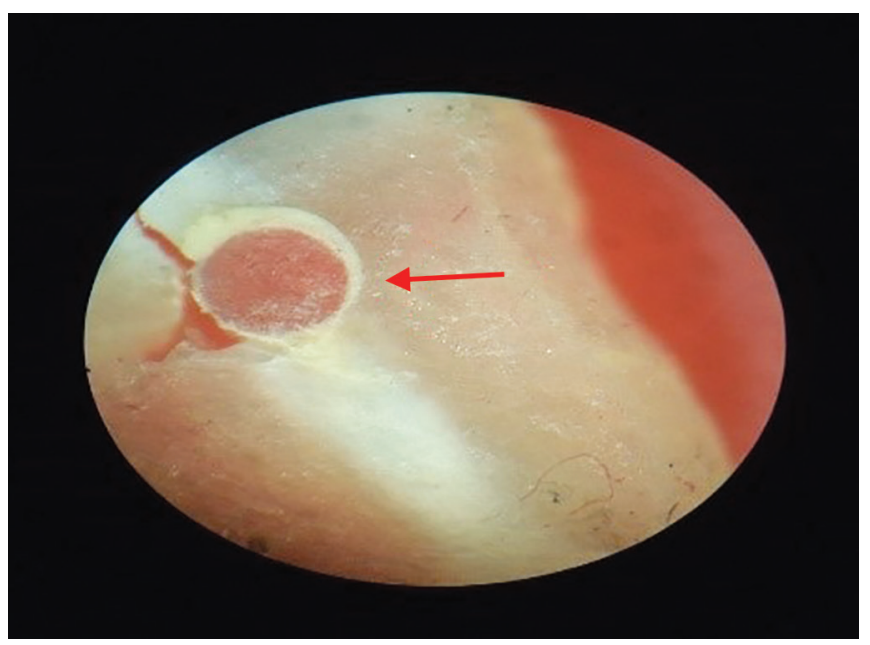

Fig. 3: Cohesive failure

Cohen et al. found that epoxy resin in the composition of resin-based cement such as $\mathrm{AH}$ Plus did not interfere with free radical activation in composite resin. As a result, resin-based sealers have no reversible action on resin cement adherence. ${ }^{23}$

According to Forough Reyhani et al., the greater bond strength of resin-based sealers is due to better and more homogeneous 


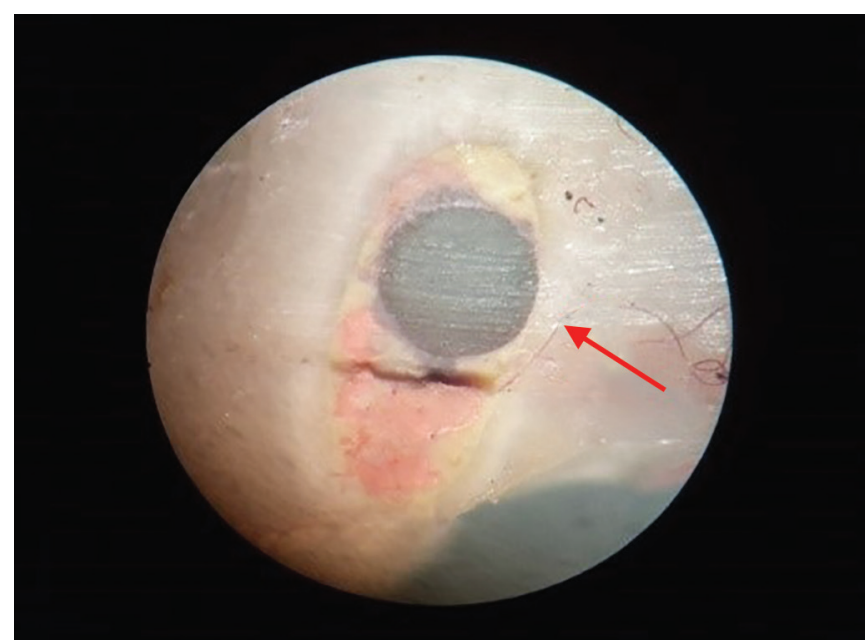

Fig. 4: Mixed failure

penetration into dentinal tubules and bonding with collagen matrix. ${ }^{24}$

The failure mode most commonly seen in the $\mathrm{AH}$ Plus group was cohesive in character, which is similar to the findings of Eldeniz et al., ${ }^{25}$ Shokouhinejad et al., ${ }^{26}$ and Vilanowa et al. ${ }^{27}$

Bio-C sealer is a calcium phosphate, calcium silicate, zirconium oxide, and calcium hydroxide-based insoluble, radiopaque, and aluminum-free substance. ${ }^{28}$ Calcium phosphate improves bioceramic setting characteristics and results in a chemical composition and crystalline structure comparable to that of tooth and bone apatite materials, increasing sealer-to-root dentin bonding. Mechanical interlocking connections are formed as the sealer particles diffuse into the dentinal tubules.

The mineral content of the sealer infiltrates the intertubular dentin, resulting in the formation of a mineral interaction zone produced after denaturing the collagen fibers with a strong alkaline sealer. There is little to no information on the effect of Bio- $C$ sealer on the failure mode of fiber posts luted with self-adhesive resin cement.

The current study found that the Bio- $C$ sealer group had more mixed type failures than the other two groups, suggesting that the Bio- $C$ sealer had no effect on the bonding of fiber post to radicular dentin; however, additional research is needed to prove this.

It is made of monobasic calcium phosphate, which facilitates the reaction with calcium hydroxide to produce water and hydroxyapatite when the sealer is activated by water. Hydroxyapatite coprecipitates with calcium silicate hydrate to form a composite-like structure that reinforces the set cement. ${ }^{29}$

Furthermore, the nanofiller in this sealer can improve bond strength. As a result, including nanosize fillers into root sealers is recommended to enhance bonding between the sealer and the root dentine. As a result, the $\mathrm{BC}$ sealer group has a higher rate of mixed failure than the other two groups. ${ }^{30}$

MTA Fillapex is a root canal sealant based on MTA and salicylate resin. It is aimed to have a high-flow rate and a thin film thickness to allow for easy penetration into lateral and accessory canals. The bond strength of MTA Fillapex is significantly smaller than that of AH Plus and Bio-C sealer.

The lower bond strength of MTA Fillapex can be explained by the higher percentage of gap-containing regions observed due to MTA
Fillapex lower adaptation to canal walls because sealers containing salicylate in the composition exhibit initial volumetric shrinkage during the setting reaction, increasing the contraction factor. On the contrary, epoxy resin sealers (AH Plus) are considered to have low contraction factor and some degree of expansion during the setting reaction as in EndoSequence BC sealer. The resin content in MTA Fillapex sealer is less as compared to AH Plus, which might also be one of the reasons why MTA Fillapex showed more number of adhesive failures as compared to the other two groups. ${ }^{31,32}$

\section{Conclusion}

The current study, as well as earlier studies, shows that sealers have an effect on the bond strength of the self-adhesive resin cement used to bind fiber post to radicular dentin. As a result, it is essential to select a sealer that will not interfere with the bond strength of the fiber post luted to the radicular dentin when using self-adhesive resin cement.

\section{References}

1. Teixeira C, Pasternak-Junior B, Borges A, et al. Influence of endodontic sealers on the bond strength of carbon fiber posts. J Biomed Mater Res Part B Appl Biomater 2008;84B(2):430-435. DOI: 10.1002/jbm.b.30888.

2. Alfredo $E$, Souza E, Marchesan M, et al. Effect of eugenol-based endodontic cement on the adhesion of intraradicular posts. Braz Dent J 2006;17(2):130-133. DOI: 10.1590/s0103-64402006000200009.

3. Morgano S, Rodrigues A, Sabrosa C. Restoration of endodontically treated teeth. Dent Clin N Am 2004;48(2):397-416. DOI: 10.1016/ j.cden.2003.12.011.

4. Bateman G, Ricketts D, Saunders W. Fibre-based post systems: a review. Br Dent J 2003;195(1):43-48. DOI: 10.1038/sj.bdj.4810278.

5. Stewardson D. Non-metal post systems. Dent Update 2001;28(7): 326-336. DOI: 10.12968/denu.2001.28.7.326.

6. Cecchin D, Farina A, Souza M, et al. Effect of root canal sealers on bond strength of fibreglass posts cemented with self-adhesive resin cements. Int Endod J 2011;44(4):314-320. DOI: 10.1111/j.13652591.2010.01831.x

7. Schwartz R, Robbins J. Post placement and restoration of endodontically treated teeth: a literature review. J Endod 2004;30(5):289-301. DOI: 10.1097/00004770-200405000-00001.

8. Shokouhinejad N, Gorjestani H, Nasseh A, et al. Push-out bond strength of gutta-percha with a new bioceramic sealer in the presence or absence of smear layer. Austr Endod J 2011;39(3):102-106. DOI: 10.1111/j.1747-4477.2011.00310.x.

9. Pawar S, Pujar M, Makandar S. Evaluation of the apical sealing ability of bioceramic sealer, AH plus and epiphany: an in vitro study. J Conserv Dent 2014;17(6):579. DOI: 10.4103/0972-0707.144609.

10. Tyagi S, Tyagi P, Mishra P. Evolution of root canal sealers: an insight story. Eur J Gen Dent 2013;2(3):199. DOI: 10.4103/ 2278-9626.115976.

11. Versiani M, Carvalho-Junior J, Padilha M, et al. A comparative study of physicochemical properties of $\mathrm{AH}$ Plus $^{\mathrm{TM}}$ and Epiphany ${ }^{\mathrm{TM}}$ root canal sealants. Int Endod J 2006;39(6):464-471. DOI: 10.1111/j.13652591.2006.01105.x.

12. DeLong C, He J, Woodmansey K. The effect of obturation technique on the push-out bond strength of calcium silicate sealers. J Endod 2015;41(3):385-388. DOI: 10.1016/j.joen.2014.11.002.

13. Gurgel-Filho E, Leite F, Lima J, et al. Comparative evaluation of pushout bond strength of a MTA-based root canal sealer. Braz J Oral Sci 2014;13(2):114-117. DOI: 10.1590/1677-3225v13n2a07.

14. Sikri V, Sidhu B, Sroa R, et al. Apical sealing ability of two novel root canal sealers: an ex-vivo study. J Int Clin Dent Res Org 2014;5(1):9. DOI: 10.4103/2231-0754.134130.

15. Barbizam J, Trope M, Tanomaru-Filho M, et al. Bond strength of different endodontic sealers to dentin: push-out test. J Appl Oral Sci 2011;19(6):644-647. DOI: 10.1590/S1678-77572011000600017. 
16. Gillen B, Looney S, Gu L, et al. Impact of the quality of coronal restoration versus the quality of root canal fillings on success of root canal treatment: a systematic review and meta-analysis. J Endod 2011;37(7):895-902. DOI: 10.1016/j.joen.2011.04.002.

17. Santos-Filho P, Veríssimo C, Soares $P$, et al. Influence of ferrule, post system, and length on biomechanical behavior of endodontically treated anterior teeth. J Endod 2014;40(1):119-123. DOI: 10.1016/ j.joen.2013.09.034.

18. Macedo V, Faria e Silva A, Marcondes Martins L. Effect of cement type, relining procedure, and length of cementation on pull-out bond strength of fiber posts. J Endod 2010;36(9):1543-1546. DOI: 10.1016/ j.joen.2010.04.014.

19. Aleisa K. The effect of different root canal sealers on the bond strength of titanium ParaPosts luted with two cements. King Saud Univ J Dent Sci 2013;4(2):65-70. DOI: 10.1016/j.ksujds.2013.03.001.

20. Rahimi S, Ghasemi N, Shahi S, et al. Effect of blood contamination on the retention characteristics of two endodontic biomaterials in simulated furcation perforations. J Endod 2013;39(5):697-700. DOl: 10.1016/j.joen.2013.01.002.

21. Sarkar N, Caicedo R, Ritwik P, et al. Physicochemical basis of the biologic properties of mineral trioxide aggregate. J Endod 2005;31(2):97-100. DOI: 10.1097/01.don.0000133155.04468.41.

22. Chadgal S, Choudhary A, Thapa T. Effect of different root canal sealers on push out bond strength of fiber posts to root canal dentin: an in-vitro study. Int J Res Health Allied Sci 2019;5(1):117-120. DOI: 10.22037/iej.v13i1.17998.

23. Cohen BI, Volovich Y, Musikant BL, et al. The effects of eugenol and epoxy-resin on the strength of a hybrid composite resin. J Endod 2002;28(2):79-82. DOI: 10.1097/00004770-200202000-00007.

24. Forough Reyhani M, Ghasemi N, Rahimi S, et al. Push-out bond strength of dorifill, epiphany and MTA-fillapex sealers to root canal dentin with and without smear layer. Iran Endod J 2014;9(4):246-250. PMID 25386203; PMCID: PMC4224760.

25. Eldeniz AU, Erdemir A, Belli S. Shear bond strength of three resin based sealers to dentin with and without the smear layer. J Endod 2005;31(4):293-296. DOI: 10.1097/01.don.0000140577.99708.c8.

26. Shokouhinejad N, Gorjestani H, Nasseh AA, et al. Push-out bond strength of gutta-percha with a new bioceramic sealer in the presence or absence of smear layer. Aust Endod J 2013;39(3):102-106. DOI: 10.1111/j.1747-4477.2011.00310.x.

27. Vilanowa WV, Carvalho-Junior JR, Alfredo E, et al. Effect of intracanal irrigants on the bond strength of epoxy resin based and methacrylate resinbased sealers to root canal walls. Int Endod J 2012;45(1):42-48. DOI: 10.1111/j.1365-2591.2011.01945.x.

28. Zhang W, Li Z, Peng B. Ex vivo cytotoxicity of a new calcium silicatebased canal filling material. Int Endod J 2010;43(9):769-774. DOI: 10.1111/j.1365-2591.2010.01733.x.

29. Loushine BA, Bryan TE, Looney SW, et al. Setting properties and cytotoxicity evaluation of a premixed bioceramic root canal sealer. J Endod 2011;37(5):673-677. DOI: 10.1016/j.joen.2011.01.003.

30. Kim J, Cho B, Lee I, et al. Effect of the hydrophilic nanofller loading on the mechanical properties and the microtensile bond strength of an ethanol-based one-bottle dentin adhesive. J Biomed Mater Res Part B 2005;72B(2):284-291. DOI: 10.1002/jbm.b.30153.

31. Amoroso-Silva PA, Guimaraes BM, Marciano MA, et al. Microscopic analysis of the quality of obturation and physical properties of MTA Fillapex. Micros Res Tech 2014;77(12):1031-1036. DOI: 10.1002/ jemt.22432.

32. Ostravik D, Nordahl I, Tibballs JE. Dimensional change following setting of root canal sealer-materials. Dent Mater 2001;17(6):512-519. DOI: 10.1016/s0109-5641(01)00011-2. 